\title{
Inertia-Free Thermally Driven Domain-Wall Motion in Antiferromagnets
}

\author{
Severin Selzer, Unai Atxitia, Ulrike Ritzmann, Denise Hinzke, and Ulrich Nowak \\ Department of Physics, University of Konstanz, D 78457 Konstanz, Germany
}

\begin{abstract}
Domain wall motion in antiferromagnets triggered by thermally induced magnonic spin currents is studied theoretically. It is shown by numerical calculations based on a classical spin model that the wall moves towards the hotter regions, as in ferromagnets. However, for larger driving forces the so called Walker breakdown which usually speeds down the wall is missing. This is due to the fact that the wall is not tilted during its motion. For the same reason antiferromagnetic walls have no inertia and, hence, no acceleration phase leading to higher effective mobility.
\end{abstract}

The interest in antiferromagnetic and ferrimagnetic materials has increased recently for several reasons. One is the more complex spin structures which lead to additional spin wave modes with higher frequencies and, consequently, faster spin dynamics than in ferromagnets (FMs). Possible applications are in the field of ultrafast spin dynamics [1,2]. Also, ferrimagnets and antiferromagnets (AFMs) have attracted a lot of attention as low-damping, insulating magnets in the emerging field of spincaloritronics [3-5] which is on the combined transport of spin and heat. Finally, antiferromagnets are also discussed as future material for antiferromagnetic spintronics, since it has been shown that despite their lack of a macroscopic magnetization their magnetic state can be controlled via spin torque switching and can be read out via their magnetoresistive properties [6]. Spintronic phenomena call for exploitation in devices with magnetic storage functionalities, where a magnetic nanostructure has to be controlled efficiently and fast. The information can be stored in magnetic domains, in isolated magnetic nanoparticles, or even in domain walls (DWs) [7]. For the latter case synthetic AFMs have been shown to pave a new road towards higher DW mobility [8].

For a ferromagnetic system, in Ref. [9] the existence of thermally driven domain-wall motion in temperature gradients was demonstrated by computer simulations based on different approaches, an atomistic spin model as well as a micromagnetic model based on the Landau-Lifshitz-Bloch (LLB) equation of motion. A thermodynamic explanation for this kind of DW motion rests on the minimization of the free energy of the DW (or the maximization of entropy). For a DW at finite temperature, the free energy is $\Delta F(T)=\Delta U-T \Delta S$, where $\Delta U$ is the internal energy and $\Delta S$ the entropy of the DW. It is a monotonically decreasing function of temperature [9-11]. This rather general argument explains a DW motion towards the hotter parts of the sample where the free energy is lower [11-13] and it can be expected to hold for other magnetic textures as well. Furthermore, it has been shown by Schlickeiser $e t$ al. that the DW motion is caused by a so-called entropic torque. The exchange stiffness is weaker for higher temperature and therefore, an effective torque on the DW is created driving it towards the hotter region [11].

A more microscopic explanation for DW motion in temperature gradients rests on the continuous stream of thermally excited magnons from the hotter towards the colder region with a transfer of angular momentum pushing the wall in the direction opposite to the magnonic spin current [14]. Theoretical investigations based on these arguments show that the magnonic torque should be analogous to the macroscopic entropy torque [15].

Like ferromagnets, antiferromagnets are materials with magnetic long-range order, but while neighboring atomic magnetic moments are aligned parallel in a FM, in an AFM they are antiparallel and compensate each other. Thus, there is no net magnetization. The antiferromagnetic order parameter, which is referred to as staggered magnetization, is hence defined as $2 \mathbf{n}=\mathbf{m}_{1}-\mathbf{m}_{2}$, where $\mathbf{m}_{1,2}$ are the magnetizations of the sublattices.

Although an AFM differs from a FM on a microscopic level, their thermodynamic equilibrium properties, as, e.g., the free energy, are the same. This is due to the fact that these properties result solely from the Hamiltonian of the system. For the simple case of a two-sublattice AFM with only nearest-neighbor interaction the identical transformation to a model with reversed sign of the exchange constant $J$ and of all spins in one sublattice leads directly to a ferromagnetic model. Accordingly, the temperature dependence of the exchange stiffness should be identical, which suggests that antiferromagnetic DWs might behave as ferromagnetic ones. Note, however, that the argument above is solely classical and quantum mechanical corrections may lead to additional effects where equilibrium properties of FMs and AFMs deviate.

On the other hand, the dispersion relations of the spin waves in FM and AFM are clearly different. In particular, in an AFM there exist circularly and linearly polarized spin waves $[16,17]$. Based on a microscopic spin wave model, it 
was argued recently that the interaction of spin waves with DWs in AFMs - and consequently their dynamics - should be distinct from the DW dynamics in FMs. The mechanism of DW motion driven by antiferromagnetic spin waves should at the microscopic level vary with the excited spin wave mode: while circularly polarized spin waves carrying angular momentum cause the DW to precess and get reflected pushing the DW away from the source, linearly polarized spin waves pass through the wall without reflection dragging the DW towards the source $[17,18]$. Following these arguments a rather complex behavior of AFM DWs could be expected.

In this Letter, we study the thermally driven DW motion in AFMs using an atomistic approach by solving the stochastic Landau-Lifshitz-Gilbert equation of motion for an atomistic spin model. Comparing our results with the DW dynamics in FMs we find similarities as well as clear differences. For small temperature gradients the wall velocity in AFMs is identical to the one in FMs while for larger driving forces the so-called Walker breakdownwhich usually speeds down the DW-is missing. Furthermore, the DW is not tilted during its motion so that it has no inertia and, hence, no acceleration phase leading to higher effective mobility.

For our study we first simulate the dynamics of a DW in a thermal gradient based on a model of classical spins in the form of normalized magnetic moments $\mathbf{S}_{i}=\boldsymbol{\mu}_{i} / \mu_{s}$ on a simple cubic lattice. We consider isotropic Heisenberg exchange interactions for nearest neighbors and a biaxial anisotropy. The Hamiltonian reads

$$
\mathcal{H}=-J \sum_{\langle i, j\rangle} \mathbf{S}_{i} \mathbf{S}_{j}-d_{x} \sum_{i}\left(S_{i}^{x}\right)^{2}-d_{z} \sum_{i}\left(S_{i}^{z}\right)^{2},
$$

with $J<0$ for antiferromagnetic order. Here $d_{z}>d_{x}>0$ are anisotropy constants defining an easy axis in the $z$ direction and an intermediate axis in the $x$ direction.

The dynamics of the normalized magnetic moments at finite temperature are given by the stochastic LandauLifshitz-Gilbert equation,

$$
\frac{\partial \mathbf{S}_{i}}{\partial t}=-\frac{\gamma}{\mu_{s}\left(1+\alpha^{2}\right)} \mathbf{S}_{i} \times\left[\mathbf{H}_{i}+\alpha\left(\mathbf{S}_{i} \times \mathbf{H}_{i}\right)\right],
$$

where $\gamma$ is the gyromagnetic ratio and $\mathbf{H}_{i}=-\partial \mathcal{H} /$ $\partial \mathbf{S}_{i}+\zeta_{i}(t)$. The first term represents a precession of the magnetic moments around an effective field $\mathbf{H}_{i}$, while the second includes phenomenological relaxation with damping constant $\alpha[19,20]$. The temperature in this model is included within the framework of Langevin dynamics by an additional white noise term $\zeta_{i}(t)$ in the effective field [21,22].

The simulations are based on the numerical integration of the stochastic Landau-Lifshitz-Gilbert equation using Heun's method [22] with time step $\Delta t=1.76 \times 10^{4} \mu_{s} /(\gamma \mathrm{J})$. We simulate an elongated three-dimensional model with
$16 \times 16 \times 256$ spins featuring open boundary conditions and an easy axis with $d_{z}=0.1 \mathrm{~J}$ while $d_{x}$ is varied. After initialization and relaxation of the DW, a linear thermal gradient is applied to the system, where the temperature increases along the $z$ direction from $T=0$ to $T=0.57 T_{c} / a$, where $T_{c}$ is the critical temperature and $a$ the lattice parameter. The damping constant is set to $\alpha=0.01$ and absorbing boundary conditions are introduced at the cold end to avoid spin wave reflection.

We start with a comparison of thermally driven DW motion in ferromagnetic and antiferromagnetic systems (Fig. 1). The first result we obtain is that in both systems the DW moves towards the hotter region, as expected from the thermodynamic considerations. But there is a significant difference in the behavior of the DW during its motion. The dynamics of the ferromagnetic DW profile reveals an acceleration phase during which the DW is forced out of its easy $x-z$ plane. This tilting depends strongly on the anisotropy $d_{x}$, the damping parameter $\alpha$, and the strength of the thermal gradient. After that acceleration the DW moves with constant velocity and constant tilting angle. For larger gradients eventually the wall starts to rotate while it is moving and the velocity decreases (see Fig. 4). This is the so-called Walker breakdown, which was observed for field-, current- and also thermally driven DW motion in FMs [9,23-25].

In case of the AFM there is no acceleration phase and no tilting of the plane containing the DW. Instead, the wall remains in its initial plane and moves with
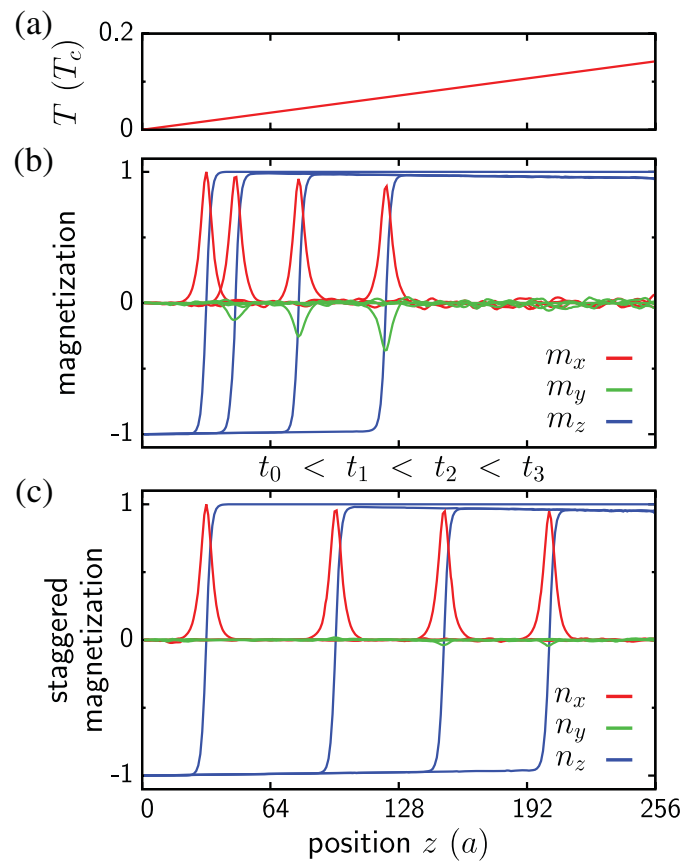

FIG. 1. Motion of the DW in a thermal gradient. Linear temperature gradient $\Delta T / \Delta z=0.56 \times 10^{-3} T_{c} / a$ (a), and pro file of the DW at different times in a ferromagnetic (b), and antiferromagnetic (c) system with $d_{x}=0.02 \mathrm{~J}$. 


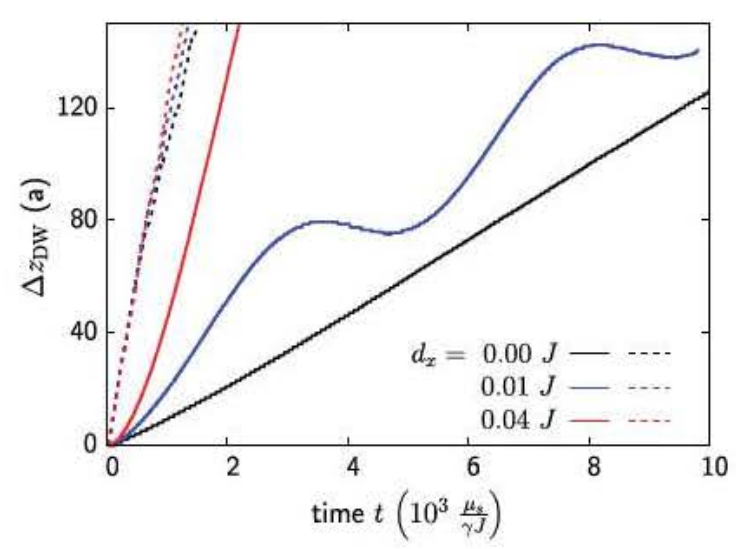

FIG. 2. Displacement of the DW versus time. Comparison between ferromagnetic (solid) and antiferromagnetic (dashed) systems with different intermediate anisotropies $d_{x}$ in a thermal gradient of $\Delta T / \Delta z=1.67 \times 10^{-3} T_{c} / a$.

constant velocity. Note that the movement of the DW in Fig. 1 shows slight deviations from a pure ballistic motion with constant velocity due to an additional diffusive component.

The tilting (or the lack of it) is crucial for the dynamics of the DW as can be seen in Fig. 2. For the ferromagnetic system three cases can be distinguished. In the case of high anisotropy $d_{x}=0.04 \mathrm{~J}$, the motion of the DW after the acceleration phase is linear and the velocity is maximal. In the case of a smaller anisotropy of $d_{x}=0.01 \mathrm{~J}$, the DW motion is accompanied by oscillations that are typical for the Walker breakdown, where the DW starts to precess and the mean velocity decreases. For $d_{x}=0$ there is no favored orientation of the wall and the DW rotates continuously and moves constantly with reduced velocity. The DW motion in the AFM, however, is independent of the anisotropy. There is no tilting of the DW and therefore no precession of the DW and no Walker breakdown. The DW mobility is much higher in AFMs for large driving forces above the Walker breakdown.

The absence of a Walker breakdown results from the symmetry of the torques acting on the DW. In a temperature gradient the exchange stiffness (the thermodynamic average of the microscopic exchange interaction) is space dependent and decreases with increasing temperature, vanishing at the Curie point. Considering the central plane of the DW the space dependent exchange field results in two torques following from the equation of motion [Eq. (2)], one contribution (the double cross product) which drives the wall and one contribution (the cross product) which-in a FM-would tilt the wall (see Fig. 3). In an AFM the first term is antisymmetric, pointing in an opposite direction on the two different sublattices, as it is necessary to drive the DW. The second torque, however, is symmetric pointing into the same direction on the two sublattices. In a FM it would tilt the wall but in an AFM these two contributions cancel, which explains the fact that

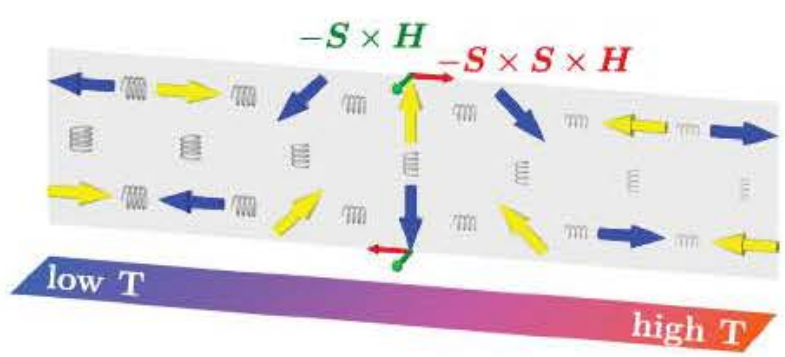

FIG. 3. Sketch of the torques acting on the central plane of a DW in a thermal gradient. The two sublattices of the AFM are occupied with blue and yellow spins, respectively.

the DW slides easily without leaving the easy plane. The DW in an AFM is displaced but not tilted in a temperature gradient. Consequently, it has no inertia and no Walker breakdown occurs.

As Fig. 4 shows, the DW motion in an AFM is not affected by an intermediate anisotropy $d_{x}$, since the wall always stays in its easy plane. The velocity of the wall is then always proportional to the temperature gradient. These findings are in clear contrast to the movement of the DW in the FM, where the velocity collapses at a certain point, the Walker breakdown. The position of the breakdown could
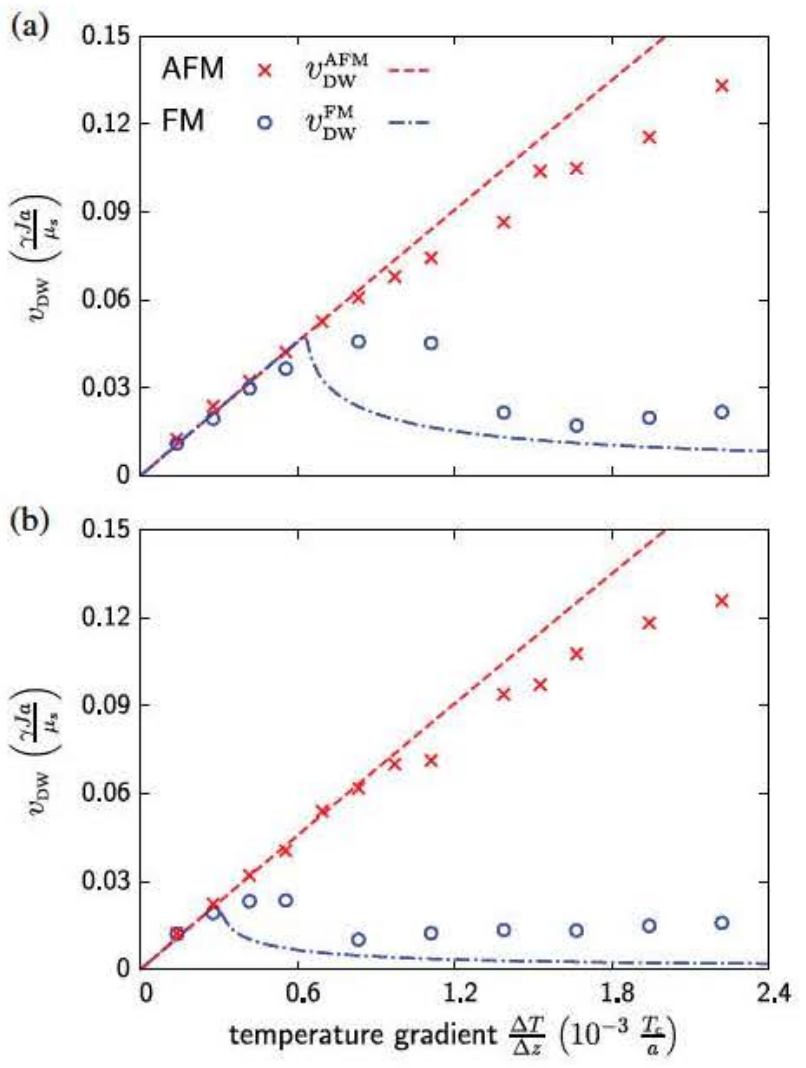

FIG. 4. DW velocity as a function of temperature gradient in an antiferromagnetic (red) and a ferromagnetic (blue) system with (a) $d_{x}=0.02 \mathrm{~J}$ and (b) $d_{x}=0.01 \mathrm{~J}$. Comparison between numerical results (points) and analytical estimates (lines). 
not be determined exactly, since close to the breakdown the precession of the DW is very slow and the wall can reach the end of the system before rotating once. Hence, the velocity seems to level off before collapsing, which is a numerical effect.

Since below the Walker threshold the wall velocities in FMs and AFMs agree a comparison with earlier results for DW motion in FMs appears useful. Schlickeiser et al. [11] derived an analytical estimation of the DW velocity and Walker threshold for DW motion in a temperature gradient within the framework of the micromagnetic LandauLifshitz-Bloch (LLB) equation in the one-dimensional limit. For the magnitude of the DW velocity they obtained

$$
\begin{aligned}
v_{\mathrm{DW}}= & \frac{2 \gamma}{M_{s}} \frac{1}{\alpha_{\perp}}\left|\frac{\partial A}{\partial z}\right|\left(1+\frac{\alpha_{\perp}^{2}}{m_{e}^{2}}\right) \\
& -\frac{\gamma \delta_{w} m_{e}(1-k)}{2 \tilde{\chi}_{\perp}} \sqrt{\left(\frac{\partial A / \partial z}{\tilde{A}_{w}}\right)^{2}-1 .}
\end{aligned}
$$

Here, the first line describes the linear increase of $v_{\text {DW }}$ while the second line, related to a rotation of the wall, contributes only above the Walker threshold $\tilde{A}_{w}$, which is given by

$$
\tilde{A}_{w}=\frac{m_{e} M_{s} \alpha_{\perp} \delta_{w}(1-k)}{4 \tilde{\chi}_{\perp}} .
$$

$M_{s}$ is the saturation magnetization (at zero temperature), while the micromagnetic exchange stiffness $A(T)$, the transverse susceptibility $\tilde{\chi}_{\perp}(T)$, the transverse damping parameter $\alpha_{\perp}(T)$, and the reduced equilibrium magnetization $m_{e}(T)$ are temperature dependent, thermodynamic equilibrium quantities. The factor $k=1-d_{x} / d_{z}$ breaks the symmetry in the $x-y$ plane.

Regarding an AFM we can neglect the second term; hence, we obtain

$$
v_{\mathrm{DW}}^{\mathrm{LLB}}=\frac{2 \gamma a^{3}}{\mu_{s} \alpha} \frac{\partial T}{\partial z} \frac{\partial A}{\partial T},
$$

with $\alpha_{\perp}=\alpha, M_{s}=\mu_{s} / a^{3}$, and atomic distance $a$. The temperature dependence of the transverse damping constant within the LLB approach is weak and neglected here as well as the term quadratic in $\alpha_{\perp}$. To include the temperature dependence of the micromagnetic exchange stiffness a simple, linear estimate is $A(T)=A(0) / T_{c}$, where it is $A(0)=J / 2 a$ in our spin model. This approximation was used in [11]. In the following, we use a better low temperature approximation which was calculated with the classical spectral density method [26,27].

There, it was found that the micromagnetic exchange $A(T)$ scales with the equilibrium magnetization $m_{e}(T)$, $A\left(m_{e}\right)=A(0) m_{e}^{2} \mathcal{D}\left(m_{e}\right)$, where $m_{e}^{2}$ dependence comes out from the free magnon gas approximation (equivalent to the mean field approximation), and $\mathcal{D}\left(m_{e}\right)$ stands for a small correction coming from the magnon-magnon interactions. At low to intermediate temperatures, one can use the wellknown low temperature relation for the equilibrium temperature, $m_{e}=1-\Omega$, where $\Omega=\mathcal{W} k_{B} T / J_{0}$ is the thermally averaged spin wave occupation defined by the Watson geometric sum, $\mathcal{N} \mathcal{W}=\sum_{\mathbf{k}} 1 /\left(1-\gamma_{\mathbf{k}}\right)$, and the exchange interaction, $J_{0}=z J, z$ being the number of nearest neighbors and $\mathcal{N}$ the number of spins in the lattice, whereas, the structure factor reads $z \gamma_{\mathbf{k}}=\sum_{\mathbf{r}_{i j}} e^{i \mathbf{k} \mathbf{r}_{i j}}$, where $\mathbf{r}_{i j}$ is the connecting to the nearest neighbor vector. As for the small correction, $\mathcal{D}\left(m_{e}\right)=1-\mathcal{G} k_{B} T / J_{0}$, where $\mathcal{G}$ is the geometrical sum, $\mathcal{N} \mathcal{G}=\sum_{\mathbf{k}} \gamma_{\mathbf{k}} /\left(1-\gamma_{\mathbf{k}}\right)$. Hence, the explicit temperature dependence of $m_{e}$ and $\mathcal{D}\left(m_{e}\right)$ can directly be used to calculate $\partial A / \partial T$, which ultimately determines the DW velocity [Eq. (5)]. We note here that both geometrical integrals, $\mathcal{W}$ and $\mathcal{G}$, depend on both, the structure of the exchange interactions and the system size through $\gamma_{\mathbf{k}}$ [28]. In the present work we use $\mathcal{W}=1.65$ and $\mathcal{G}=0.5$, related to the finite size of the atomistic system utilized in our computer simulations. Excellent agreement between our analytical estimations of the $v_{\mathrm{DW}}^{\mathrm{AFM}}$ and computer simulations is found for a range of thermal gradients, which allow us to conclude that in AFMs, as for FM, the so-called entropic torques drive the DW motion. Note, that for very high driving forces the DW velocity cannot further increase, but more likely saturates, as it should be limited by the maximum group velocity of the excited magnons $[18,29]$. This limit cannot be further explored due the finite system size.

Our discussion above rests on Eq. (3), which was derived for a FM. Though our numerical results suggest agreement with this equation a strict derivation of the DW velocity of AFMs in temperature gradients should rest on a two-sublattice version of the LLB equation [30], an effort which is beyond the scope of this work. Furthermore, our discussion above is purely classical. Taking into account quantum effects the entropic torques in ferro- and antiferromagnets are not necessarily identical. Nevertheless, their symmetry will be the same and thus our main result, the inertia-free behavior of the antiferromagnetic DW, will still hold true. The effects of monochromatic spin waves $[17,18]$ which can indeed move the AFM DW wall in either direction vanish in a thermally excited spin wave spectrum. While circularly polarized spin waves cause the DW to precess and are reflected, for linearly polarized spin waves which can be seen as a combination of right- and leftcircularly polarized spin waves there is no net rotation of the wall. In a temperature gradient right- and left-circularly polarized spin waves are excited equally. Hence, on average there is no (net) rotation of the DW and therefore no reflection.

In conclusion, DWs in antiferromagnets are driven by thermal gradients and move towards the hotter region. On a macroscopic level this is explained by the minimization of 
the free energy and agrees with older results for thermally driven DW motion in ferromagnets. However, in contrast to FMs the dynamics of the wall is independent of the intermediate anisotropy which defines the easy plane containing the wall magnetization. The reason for this is the fact that those torques which-in a FM-tilt the wall cancel each other in the two sublattices of the AFM. This has two consequences. One is the absence of the Walker breakdown in AFMs leading to a higher wall mobility. This is increasingly important in the limit of low intermediate anisotropy where DWs in FMs always must precess with low DW velocity. The other consequence of the lack of DW tilting is that DW in AFMs are massless. Because of the lack of inertia they do not accelerate but reach their stationary DW velocity instantaneously. This is very important for experiments and technical applications using pulsed driving forces, since one could operate on much shorter time scales. Furthermore, the argument of counteracting torques leading to massless DWs is not restricted to temperature gradients but also applies to other driving mechanisms in antiferromagnets featuring staggered torques, like Néel spin-orbit torques [29] or current driven DW motion in synthetic antiferromagnets [8,31].

We thank the DFG for financial support through the SFB 767 and SPP 1538. U. A. gratefully acknowledges support from EU FP7 Marie Curie Zukunftskolleg Incoming Fellowship Programme, University of Konstanz (Grant No. 291784).

[1] A. Kirilyuk, A. V. Kimel, and T. Rasing, Rev. Mod. Phys. 82, 2731 (2010.

[2] A. Kirilyuk, A. V. Kimel, and T. Rasing, Rep. Prog. Phys. 76, 026501 (2013).

[3] K. Uchida, J. Xiao, H. Adachi, J. Ohe, S. Takahashi, J. Ieda, T. Ota, Y. Kajiwara, H. Umezawa, H. Kawai, G. E. W. Bauer, S. Maekawa, and E. Saitoh, Nat. Mater. 9, 894 (2010).

[4] G. E. W. Bauer, E. Saitoh, and B. J. van Wees, Nat. Mater. 11, 391 (2012).

[5] S. R. Boona, R. C. Myers, and J. P. Heremans, Energy Environ. Sci. 7, 885 (2014).

[6] P. Wadley et al., Science, doi:10.1126/science.aab1031 (2016).

[7] S. S. P. Parkin, M. Hayashi, and L. Thomas, Science 320, 190 (2008).

[8] S. H. Yang, K. S. Ryu, and S. Parkin, Nat. Nanotechnol. 10, 221 (2015).

[9] D. Hinzke and U. Nowak, Phys. Rev. Lett. 107, 027205 (2011).
[10] D. Hinzke, N. Kazantseva, U. Nowak, O. N. Mryasov, P. Asselin, and R. W. Chantrell, Phys. Rev. B 77, 094407 (2008).

[11] F. Schlickeiser, U. Ritzmann, D. Hinzke, and U. Nowak, Phys. Rev. Lett. 113, 097201 (2014).

[12] X. S. Wang and X. R. Wang, Phys. Rev. B 90, 014414 (2014).

[13] P. Yan, Y. Cao, and J. Sinova, Phys. Rev. B 92, 100408 (2015).

[14] P. Yan, X. S. Wang, and X. R. Wang, Phys. Rev. Lett. 107, 177207 (2011).

[15] S. K. Kim and Y. Tserkovnyak, Phys. Rev. B 92, 020410 (2015).

[16] F. Keffer, H. Kaplan, and Y. Yafet, Am. J. Phys. 21, 250 (1953).

[17] E. G. Tveten, A. Qaiumzadeh, and A. Brataas, Phys. Rev. Lett. 112, 147204 (2014).

[18] S. K. Kim, Y. Tserkovnyak, and O. Tchernyshyov, Phys. Rev. B 90, 104406 (2014).

[19] D. Landau and E. Lifshitz, Phys. Z. Sowjetunion 8, 153 (1935); [Ukr. J. Phys. 53 (2008)].

[20] T. Gilbert, Phys. Rev. 100, 1243 (1955); T. L. Gilbert and J. M. Kelly, in Conf. Magnetism and Magnetic Materials, Pittsburgh, PA, 1955 (American Institute of Electrical Engineers, New York, 1955), pp. 256 263; IEEE Trans. Magn. 40, 3443 (2004).

[21] W. F. Brown, Phys. Rev. 130, 1677 (1963).

[22] U. Nowak, Handbook of Magnetism and Advanced Mag netic Materials (John Wiley \& Sons, Chichester, 2007), Vol. 2, pp. 858876.

[23] N. L. Schryer and L. R. Walker, J. Appl. Phys. 45, 5406 (1974).

[24] A. Thiaville, Y. Nakatani, J. Miltat, and Y. Suzuki, Euro phys. Lett. 69, 990 (2005).

[25] C. Schieback, D. Hinzke, M. Kläui, U. Nowak, and P. Nielaba, Phys. Rev. B 80, 214403 (2009).

[26] A. Cavallo, F. Cosenza, and L. De Cesare, in New develop ments in Ferromagnetism Research, edited by V. N. Murray (Nova Science Publischers, Inc., New York, 2005), Chap. 6, pp. 131187.

[27] R. Bastardis, U. Atxitia, O. Chubykalo Fesenko, and H. Kachkachi, Phys. Rev. B 86, 094415 (2012).

[28] U. Atxitia, D. Hinzke, O. Chubykalo Fesenko, U. Nowak, H. Kachkachi, O. N. Mryasov, R. F. Evans, and R. W. Chantrell, Phys. Rev. B 82, 134440 (2010).

[29] O. Gomonay, T. Jungwirth, and J. Sinova, Phys. Rev. Lett. 117, 017202 (2016).

[30] U. Atxitia, P. Nieves, and O. Chubykalo Fesenko, Phys. Rev. B 86, 104414 (2012).

[31] M. Kuteifan, M. V. Lubarda, S. Fu, R. Chang, M. A. Escobar, S. Mangin, E. E. Fullerton, and V. Lomakin, AIP Adv. 6, 045103 (2016). 